\title{
Suizidkandidat Hemingway
}

\section{Der alte Mann \\ und das Gewehr}

\begin{abstract}
Tiefe körperliche und psychische Wunden, deren Anzahl mit den Lebensjahren zunahm, führten zum dramatischen Ende Ernest Hemingways im Sommer 1961. Ein Ende, das in scharfem Kontrast stand zu seinem offenen Wesen und seinem Erfolg als Autor.
\end{abstract}

\begin{abstract}
Dem Neurowissenschaftler Dr. Sebastian Dieguez von der Universität Friborg in der Schweiz zufolge lassen sich eine ganze Palette von Risikofaktoren für Suizidalität finden. So gab es in der Familie Hemingway viele Suizide, die auf eine genetische Prädisposition für psychische Störungen schließen lassen. Ernest Hemingways strenger Vater litt unter starken Stimmungsschwankungen und erschoss sich 1928. Ernests Schwester Ursula und der Bruder Leicester suizidierten sich ebenfalls. Hemingways Sohn Gregory hatte eine bipolare Störung, auch eine Enkelin litt an verschiedenen neurologischen und psychischen Störungen und beging 1996 Suizid.
\end{abstract}

\section{Statt sich sellbst to̊tete er Tiere}

Das psychologische Profil Hemingways ergibt einen kompetitiven, ehrgeizigen Charakter. Er ist ein impulsiver Mensch, der häufig lügt, gerne übertreibt und sich oft kindlich und egozentrisch verhält. Zugleich fühlte er sich unzulänglich, war mit sich selbst unzufrieden. Hemingway hatte sich ein Image aufgebaut, eine Fassade teilweise brachial zur Schau gestellter Virilität, hinter der er tiefsitzende Ängste zu verbergen suchte. Alkohol, Jagen und Kämpfen im Angesicht der Gefahr sieht Dieguez als Mittel, die Aggressivität und den Drang zu Gewalttätigkeit zu kanalisieren und selbstzerstörerische Gedanken zu externalisieren: „Ich habe viel Zeit damit verbracht, Tiere und Fische zu töten, daher werde ich mich nicht selbst töten“, soll er zur US-amerikanischen Schauspielerin Ava Gardner (1922-1990) im Jahre 1954 geäußert haben.

\section{Hirnschäden durch Alkohol und Unfälle?}

Er konnte nicht gut allein sein, beständig versuchte er, sein Leben mit Reizen und Reisen, Action und Gefahr zu füllen, sei es als Kriegsberichterstatter, Großwildjäger oder als Fan von Stier- und Boxkämpfen. Seine Stimmungsschwankungen sind gut dokumentiert. Die manischen Phasen waren durch eine ungeheure Produktivität gekennzeichnet - angeblich hat er die Kurzgeschichten „Die Killer“, „Heute ist Freitag" und "Zehn Indianer" an einem Tag geschrieben. Der liebenswerte und sympathische Hemingway konnte unverhofft aggressiv zu Fremden oder selbst Freunden werden. Hinzu kam sein Alkoholismus. Die Alkoholabhängigkeit muss letztlich auch Hirn- und andere organische Schäden zur Folge gehabt haben, die zusammen mit den psychischen Beeinträchtigungen die psychotischen Symptome in seinen letzten Lebensjahren förderten. Wiederholte Schädeltraumata des Abenteurers könnten zusätzlich dazu beigetragen haben. Von 1954 an, er hatte den Literaturnobelpreis aus gesundheitlichen Gründen nicht persönlich entgegen nehmen können, litt Hemingway zunehmend unter seinen körperlichen und psychischen Gebrechen. Er entwi- ckelte Wahnideen und zeigte hypochondrischen Züge.

\section{Drei Suizidversuche innerhalb von vier Tagen}

Im November 1959 wurde er für insgesamt sieben Wochen in die Mayo Clinic in Rochester, Minnesota, aufgenommen und mit Elektrokrampftherapie behandelt. Diese schadete ihm jedoch eher, da sie den aufgrund des Alkoholabusus, der Depression und den Kopftraumata sowieso bereits fortschreitenden Gedächtnisverlust noch verstärkte. Hemingway muss sich terminal krank gefühlt haben. Im April 1961 unternahm er innerhalb von vier Tagen drei Suizidversuche. Zweimal wollte er sich erschieBen, einmal kam er einem sich drehenden Propeller des Flugzeugs, das ihn erneut nach Rochester bringen sollte, gefährlich nahe. Warum er am 26. Juni 1961 trotz der offensichtlichen Geisteskrankheit und Suizidalität entlassen wurde, ist unklar. Hemingway wollte freilich nicht in Rochester bleiben, dort zerstöre man sein Gehirn. In einer fünftägigen Autofahrt wurde er nach Hause gebracht und traf am 30. Juni in Ketchum, Idaho, ein. Am 2. Juli 1961, wenige Tage vor seinem 62. Geburtstag, feuerte er zwei Gewehrpatronen gleichzeitig in seinen Schädel ab.

(Thomas Meißner)

Den ungekürzten Beitrag samt Literaturliste finden Sie im Dossier "Prominente Patienten“ auf www.springermedizin.de 